\title{
Robotic Adaptations for Building Works; assembly of concrete blocks "stay-in-place" with robots
}

\author{
Paula Ignacia Ulloa Aguayo \\ Universidad del Bío Bío | Chile | pulloa@ubiobio.cl \\ Rodrigo García-Alvarado \\ Universidad del Bío-Bío | Chile | rgarcia@ubiobio.cl \\ Mauricio Osses Coloma \\ Chile | mauosses@udec.cl \\ Alexis Pérez Fargallo \\ Universidad del Bío Bío | Chile | aperezf@ubiobio.cl
}

\begin{abstract}
The progress fostered by the fourth industrial revolution requires building methods according to productivity and sustainability, which also considers the human factor. Therefore, this work addresses safety and efficiency of construction tasks and transformation by robots. As a case study, assembly process of "stay-in-place" concrete blocks is studied, consisting of prefabricated insitu molding pieces with thermal insulation, and finishing included. The movements of the worker in the assembly are evaluated, to be supported by robot procedure and its implications in architectural design. By implementing this technology, human risks in the execution of a work are reduced, allowing greater constructive productivity.
\end{abstract}

Keywords: Robots in Architecture; Building Works; Assembly; Digital Fabrication.

\section{INTRODUCCIÓN}

El acelerado progreso que propicia la cuarta revolución industrial requiere de métodos constructivos acorde a las necesidades de productividad y sustentabilidad (CORFO 2015), que considere además al factor humano como agente ejecutor. Por lo que, este trabajo aborda una problemática que surge desde la necesidad de seguridad y eficiencia ante factores de riesgo asociado a faenas constructivas y como éstas enfrentan su transformación hacia labores facilitadas por robots (Bock, 2015).

Como caso se estudia el proceso de montaje del sistema constructivo "stay-in-place", consistente en bloques de prefabricados de concreto como moldaje permanente, con aislación térmica, y terminación final incluida (De Sutter et al, 2014). Se evalúan los movimientos del trabajador en el montaje, logrando identificar maniobras claves para ser apoyadas por la robótica y sus implicancias en el diseño arquitectónico (Sousa, 2017). Al implementar esta tecnología, se pretende reducir los riesgos laborales en la ejecución de una obra, permitiendo una mayor productividad y seguridad constructiva (Garcia et al, 2019).

La robótica, como automatización de procesos, ha abierto diversas posibilidades en la arquitectura y construcción, tanto en nuevas capacidades expresivas, como efectividad de la ejecución (Gramazio y Kohler, 2014; Dagmar et al, 2016). La integración de sistemas de diseño BIM y fabricación digital para la construcción ha abierto mayormente el espectro de posibilidades, al otorgar control y flexibilidad (Davtalab et al, 2018). Sin embargo, escasa atención se ha brindado a las labores de construcción y sus condiciones de seguridad. La convivencia de faenas humadas y automatizadas en la edificación implica regular y focalizar esfuerzos en complementar estas condiciones mediante la utilización de nuevos equipos robóticos. En el desarrollo de nuevos sistemas constructivos, esta interrelación es crucial para determinar una estrategia de implementación segura y efectiva, pero también para advertir sus implicancias en la gestión de la edificación y en el diseño arquitectónico.

\section{SISTEMA CONSTRUCTIVOS "STAY-IN PLACE"}

La construcción, operación y mantenimiento de los edificios consume un alto porcentaje de la energía disponible, siendo de alrededor de $30-40 \%$ a nivel mundial, de acuerdo a diversas estimaciones (PérezLombard, Ortiz \& Pout, 2008; Comstock Maggie \& Garrigan Curt, 2012). En el caso de Chile, no se dispone de datos tan fiables, pero se calcula que los edificios del país necesitan tres veces más energía que aquellos situados en países con climas comparables (CORFO, 2015). El elevado consumo de energía de los edificios se debe a una combinación de laxos estándares edificatorios y a una industria de la construcción carente de un potente desarrollo científico y tecnológico. De acuerdo a los últimos datos disponibles del INE, alrededor de la mitad de los 18,7 millones de m2 edificados en Chile en el 2014 han sido construidos con hormigón ( $\approx 10$ millones), muy por delante de materiales como el metal ( $\approx 1,5$ millones) o la madera ( $\approx 2,2$ millones) (INE, 2014). Si bien, el hormigón armado tiene una muy buena resistencia a los terremotos, presenta dos problemas principales: requiere de un largo y costoso proceso de puesta en obra y posee una elevada conductividad térmica. 
La puesta en obra requiere el uso de moldajes, ya que el hormigón llega a la obra en un estado fluido, se vierte en un molde y se deja fraguar durante varios días (7 aproximadamente). Estos moldes se denominan encofrados y suelen dividirse en dos tipos: desmontable y perdido. Los encofrados desmontables suelen ser metálicos, se montan para recibir el hormigón fresco y se retiran al haber fraguado éste. Estos elementos deben arrendarse y suponen un coste adicional en la construcción. Los de tipo perdido suelen ser de algún material de base cementicia con una variedad de aditivos y adiciones, o de materiales cerámicos o plásticos, que reciben el hormigón fresco y no se retiran. En ambos casos, el hormigón fraguado y moldeado adquiere las propiedades estructurales necesarias, que suelen ser adecuadas para soportar el propio peso del edificio y otras cargas externas, dentro de las cuales la más importante es la sísmica. Sin embargo, un muro de hormigón armado con un espesor habitual, que suele oscilar entre $10-20 \mathrm{~cm}$, tiene unas deficientes propiedades higrotérmicas. Incluso con el máximo espesor $(20 \mathrm{~cm})$ el coeficiente $U$ resultante es de $3,41 \mathrm{w} / \mathrm{m} 2 \mathrm{~K}$, lo cual no cumple con la mayoría de los mínimos establecidos por la ley chilena y sus continuas actualizaciones que prevén límites incluso más estrictos. Por ello, se hace necesario añadir una o varias capas adicionales que otorguen al conjunto unas idóneas propiedades higrotérmicas; para esto suelen emplearse productos aislantes y/o impermeables. Estos sistemas se denominan en inglés EIFS (External insulation \& finishing system) y presentan varios problemas, siendo los principales: costo elevado; requerir varios materiales; tiempo de ejecución, ya que se necesitan diferentes operarios para instalar cada capa; además de incompatibilidad y falta de coordinación entre todos los materiales de estas, debido a las diferentes tolerancias.

\section{DESCRIPCIÓN DEL PREFABRICADO STAY IN PLACE}

La presente tecnología corresponde a un prefabricado para encofrar muros de hormigón del tipo encofrado perdido, el cual asegura las propiedades estructurales, acústicas e higrotérmicas de los muros. Este encofrado tiene una forma paralelepipédica y se compone de dos caras planas que se disponen de forma paralela. El espacio entre ambas caras se encuentra vacío para poder verter el hormigón fresco. A su vez, todo el conjunto está unificado por una armadura metálica que le otorga solidez. Las caras interior y exterior están compuestas por una o hasta 4 capas de morteros ligeros de baja conductividad térmica y una capa exterior de acabado de mortero con fibra de vidrio; estas capas se combinan dependiendo de los requerimientos de cada zona climática. Las dosificaciones empleadas en las capas tienes las propiedades descritas en la tabla 1.

Tabla 1. Propiedades de los materiales que componen las caras del prefabricado.

\begin{tabular}{lccccc}
\hline Material & $\begin{array}{c}\text { Conductividad } \\
{\left[\mathrm{W} / \mathrm{m}^{2} \mathrm{~K}\right]}\end{array}$ & $\begin{array}{c}\text { Densidad } \\
{[\mathrm{kg} / \mathrm{m} 3]}\end{array}$ & $\begin{array}{c}\text { Resist. al vapor } \\
\text { de agua }(\mu)\end{array}$ & $\begin{array}{l}\text { Permeancia al } \\
\text { Recubrimiento } \\
(\mathrm{g} / \mathrm{m} 2 \mathrm{~h} \mathrm{mmHg})\end{array}$ & $\begin{array}{c}\text { Permeabilidad } \\
\text { al vapor de agua } \\
(\mathrm{g} / \mathrm{m} 2 \mathrm{~h} \mathrm{mmHg})\end{array}$ \\
\hline Dosif. 01 & 0,038 & 232 & 7,205 & 0,350 & 0,01476 \\
Dosif. 02 & 0,059 & 333 & 11,754 & 0,213 & 0,00905 \\
Dosif. 03 & 0,081 & 430 & 21,232 & 0,118 & 0,00501 \\
Mortero & 1,000 & 1900 & 25,000 & 0,100 & 0,00425 \\
con fibra & & & & & \\
lde vidrio & & & & & \\
\hline
\end{tabular}

Por otra parte, la armadura metálica se compone de barras de acero corrugado $\mathrm{A} 440-280 \mathrm{H}$ de $4 \mathrm{~mm}$ y $6 \mathrm{~mm}$ de diámetro . Este prefabricado tiene una longitud total de 120 $\mathrm{cm}$, una altura de $40 \mathrm{~cm}$ y un espesor total entre $19-37$ $\mathrm{cm}$ y su peso varía entre $26-31 \mathrm{~kg}$ dependiendo del porcentaje de cada una de las 3 dosificaciones y de la armadura metálica. El espesor varía debido a los requerimientos estructurales y térmicos; el espesor del muro de hormigón armado del núcleo varía entre 10 - 20 $\mathrm{cm}$ y los espesores de las caras interior y exterior, entre 35 - $115 \mathrm{~mm}$; ambas caras se recubren por una última capa de mortero con un espesor de $15 \mathrm{~mm}$. De este modo, el prefabricado puede cumplir con los requerimientos estructurales e higrotérmicos de distintas zonas térmicas de Chile. En la Tabla 2 se presenta el detalle de 15 prototipos evaluados, donde se puede apreciar que el peso del prefabricado en sus diferentes configuraciones oscila entre $26-31 \mathrm{~kg}$

Tabla 2. Propiedades de Prefabricados.

\begin{tabular}{|c|c|c|}
\hline $\begin{array}{l}\text { Propiedad } \\
\text { (unidad de medición) }\end{array}$ & Valor & Técnica de Medición \\
\hline Longitud $(\mathrm{cm})$ & 120 & Medición en laboratorio \\
\hline Anchura $(\mathrm{cm})$ & $19-37$ & Medición en laboratorio \\
\hline Altura $(\mathrm{cm})$ & 40 & Medición en laboratorio \\
\hline Peso (kg) & $26,50-30.63$ & Medición en laboratorio \\
\hline \multicolumn{3}{|l|}{ Transmitancia } \\
\hline Térmica $\left(\mathrm{W} / \mathrm{m}^{2} \mathrm{~K}\right)$ & \multicolumn{2}{|c|}{$\begin{array}{l}\text { 0,28-1,1,02 Cálculo conforme a la } \\
\text { NCh 853.Of2014 }\end{array}$} \\
\hline Condensación & & \\
\hline $\begin{array}{l}\text { Superficial } \\
\text { Intersticial }\end{array}$ & NO & $\begin{array}{l}\text { Cálculo conforme a la } \\
\text { NCh1973.Of2014 y } \\
\text { NCh1973.Of87 }\end{array}$ \\
\hline $\begin{array}{l}\text { Resistencia a } \\
\text { Compresión }\end{array}$ & $1,65-2,85$ & Medición en laboratorio \\
\hline $\begin{array}{l}\text { Resistencia a } \\
\text { Flexión }\end{array}$ & $0,22-1,0$ & Medición en laboratorio \\
\hline
\end{tabular}

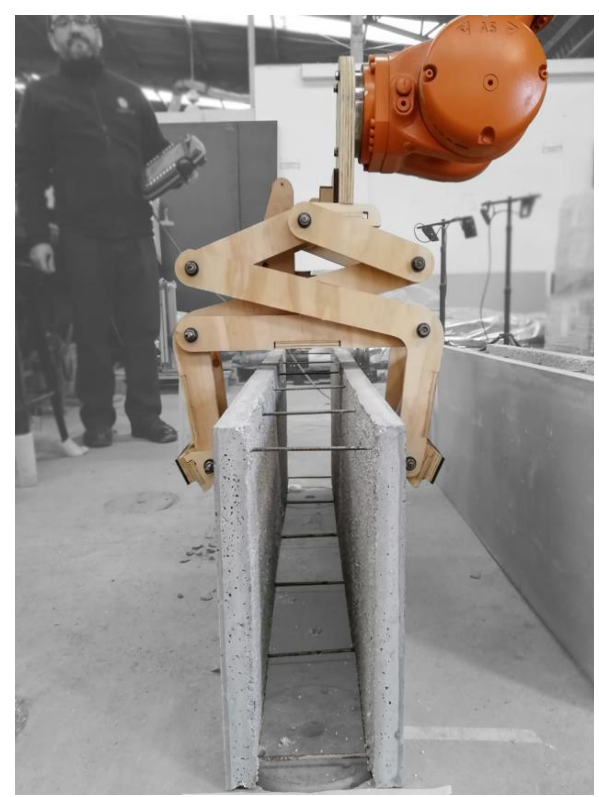

Figura 1: Bloques STAY IN PLACE. Elaboración propia. 
El producto resultante es un encofrado perdido colaborante cuyas dimensiones y peso le permiten ser manipulado por al menos dos operarios. Su resistencia a la flexión se encuentra entre $0,22-1 \mathrm{~kg} / \mathrm{cm} 2$ en función al tipo de apoyo y del prefabricado y su resistencia a la compresión entre $1,65-2,85 \mathrm{~kg} / \mathrm{m}^{2}$, lo que lo hace adecuado para soportar las presiones debidas al vertido de hormigón fresco que constituye el núcleo estructural entre ambas caras con una configuración adecuada, siempre que la altura del hormigonado no supere los dos metros en cada vertido.

\section{FACTORES HUMANOS EN LA CONSTRUCCION}

El segundo tipo de accidente que se presenta con mayor proporción en el rubro de la construcción corresponde a caídas desde distintos niveles, con un 22,7\%, predominando las caídas de altura (desde techumbres, escaleras, andamios), y por la presencia de condiciones de los pisos mojados y resbaladizos. Estos accidentes provocan diversas lesiones, fracturas y traumatismos en diferentes partes del cuerpo, las que requieren atención médica y seguimiento. (Carrasco et al, 2016)

Se considera trabajo en altura a toda actividad que se realiza por encima 1,8 metros, ejecutando trabajos sobre o bajo el suelo, como por ejemplo en sistema de plataformas fijas, móviles o rodantes, en pozos o excavaciones en general, fachadas o estructuras en voladizo. Para todos los trabajos realizados en altura, se debe utilizar arnés de seguridad con un sistema de afianzamiento (estrobo y línea de vida) independiente a la plataforma de trabajo, calculado y diseñado por un profesional competente. (Achs, Definición trabajo en altura, 2016). Considerando además en la implementación de medidas de distanciamiento social en el trabajo $>1.0 \mathrm{~m}$. por motivos de salud debido a pandemia.

\section{METODOLOGIA}

El estudio realizado contempla la evaluación de la faena de montaje para identificar en qué momento es pertinente introducir ayuda robótica en el proceso de montaje de bloques del sistema Stay in Place, estableciendo los parámetros a integrar para su implementación en la realidad. Este trabajo se realizó en 5 etapas:

\section{1.- IDENTIFICACIÓN DE LA PROBLEMÁTICA A TRAVÉS DE UN ESTUDIO ERGONÓMICO}

El estudio ergonómico hace referencia a la evaluación del factor humano y cómo este incide en el armado de muros en bloques. Se visualiza como objetivo general, el poder hacer una comparación objetiva, entre el desempeño de tres trabajadores durante la ejecución de la tarea y lo que rinde la programación de movimientos automatizados (robot) de piezas, en función de una unidad total. Teniendo como característica evitar los accidentes en manipulación y de caídas en altura. (Cervantes, Abraca. 2005)

Para enmarcar la evaluación, se define como descripción de la tarea: el armar en altura un grupo de 6 bloques montados en una línea vertical durante un ciclo continúo. Se define ciclos como el montaje continuado de 6 bloques. Este fue ejecutado por tres trabajadores, de los cuales dos realizaron la manipulación de los bloques durante su acople vertical y uno operó el montacargas como ayuda mecánica. Para el registro, es utilizaron cámara, huincha métrica, pesa digital. Se hizo una entrevista, referente a como era el proceso de armado y la sensación de los trabajadores al ejecutar la tarea. Para la evaluación bioenergética/fisiológica, Se registraron la presión arterial y la frecuencia cardiaca al inicio y al término de la tarea. Para evaluar el Manejo Manual de Cargas (MMC), se utiliza la metodología MAC Manual Handling Assessment Charts, que hace referencia al levantamiento, descenso con dos manos realizado de dos a cuatro trabajadores y transporte de cargas con una o dos manos o por uno o dos trabajadores. Método requerido por la ley No 20.001.Chile y la "Guía Técnica para la Evaluación y Control de los Riesgos Asociados al Manejo o Manipulación Manual de Carga".

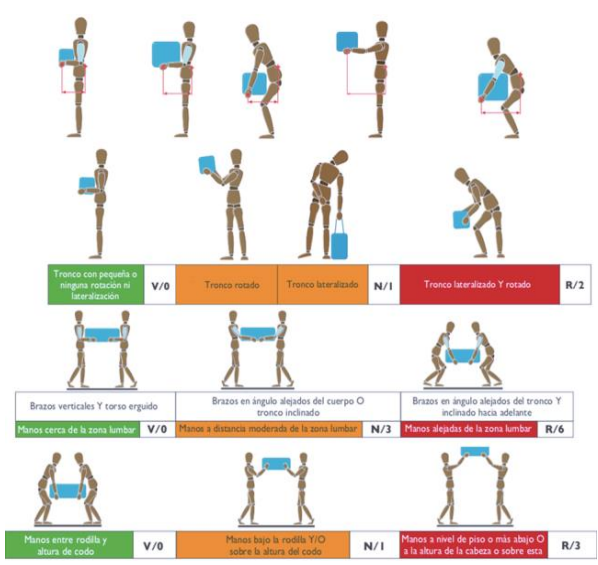

Figura 2: Referencia de la aplicación del método MAC y sus rangos. Guía Técnica para la Evaluación y Control de los Riesgos Asociados al Manejo o Manipulación Manual de Carga.

\section{2.- VIRTUALIZACIÓN DEL SISTEMA CONSTRUCTIVO, MODELACIÓN BIM DE BLQUES.}

La modelación BIM del sistema Stay in Place se realiza utilizando el software Revit. Esta plataforma trabaja la creación de Proyectos de Arquitectura a través de la articulación de diferentes archivos bases llamadas familias, cada familia representa un elemento arquitectónico o constructivo por cada familia hay tipo de elementos capaces de contener información técnica en su representación volumétrica.

Entonces, entendiendo las lógicas constructivas del sistema Stay in Place es que se propone el diseño de piezas modulares que permitan el diseño esencial de espacialidades.

\section{3.- CREACIÓN DE UNA HERRAMIENTA PARA EL ROBOT.}

El robot disponible para el estudio es un brazo robótico de $2,5 \mathrm{~m}$. de alcance movido por 6 ejes axiales a través de servomotores con precisión de 0,00000001 mm. Puede levantar en su flange (punta del brazo) un máximo de $120 \mathrm{~kg}$. alcanzando una velocidad máxima de $2,0 \mathrm{~m} / \mathrm{s}$. Gracias a sus articulaciones puede orbitar en los 3 ejes cartesianos desde su base, el punto $(0,0,0)$ del sistema; sin embargo, este alcance depende de la herramienta, del diseño de la herramienta y la técnica de movimientos que realice para utilizarla correctamente.

Para apoyar la faena de montaje de bloques Stay in Place fue necesario pensar en una herramienta que permitiera 
levantar bloques y posicionarlos como estructura vertical. En condiciones ideales para esta tarea existen pinzas robóticas que conversan con el robot con como si incorporáramos un $7^{\circ}$ eje de movimiento que integre los movimientos de la pinza al robot, sin embargo, si no contamos con estas herramientas, la creatividad surge para adecuar soluciones análogas que conversen con el movimiento de creación digital.

\section{4.- PROGRAMACIÓN DE LA SIMULACIÓN ROBÓTICA.}

Programar movimientos robóticos para la construcción implica establecer la interoperabilidad del modelo BIM del proyecto, con el simulador robótico (Grasshopper + KUKAPRC), que traduce las geometrías a coordenadas de movimiento para el robot. Relacionando datos de modelación a técnicas constructiva condicionadas al uso de una herramienta determinada en un espacio determinado, obtenemos como resultado la programación paramétrica que necesitamos para construir. Este proceso es la antesala del traspaso de información digital a la realidad que habitamos, por lo que es necesario realizar programaciones que sean flexibles, intuitivas en el ingreso de datos y manipulables para posibles modificaciones de emergencia.

De acuerdo a los estudios previos, el robot puede incorporarse en la faena de montaje de bloques tomando unidades para levantarlas, trasladarlas hasta pasar sobre la enfierradura de refuerzo, bajarlas y posicionarlas en orden como elementos constructivos del proyecto que se esté construyendo. Para esto, utilizamos un tipo de programación que integra traslado de objetos y operación de herramienta de forma simultánea, generando el orden de la trayectoria de movimientos del robot a través de una secuencia de puntos de coordenadas.

Para importar un modelo Revit a Grasshopper existen diversos plugin que ayudan en este objetivo, sin embargo, se ha optado por utilizar la menor cantidad de recursos a modo de poder adaptarse a circunstancia desfavorables.

\section{5.- TESTEOS}

Las pruebas de movimientos con el robot Kuka con pinza de izaje y bloques "stay-in-place" efectuadas en el laboratorio CITEC UBB / CIPICS.

Para que la trayectoria programada pueda utilizarse en interacción con la realidad, se establecen los siguientes parámetros de configuración:

1.- Posición de la Herramienta: TCP o punto central de la herramienta del robot. Por lo general al integrar una herramienta nueva al flange es necesario calibrar su centro para dar a entender al robot que debe mover su TCP al nuevo TCP de la herramienta, estableciendo un nuevo punto de coordinación de movimientos. Sin embargo, en este estudio se tuvo como precaución mantener el TCP que vienen calibrado por defecto (el centro del flange), ya que, al estar operando una herramienta dinámica de baja precisión, es engañoso establecer una posición TCP con exactitud, por lo tanto, se decide trabajar con el TCP propio del robot.

2.- Posición A: Coordenadas del TCP al momento de topar el primero bloque con la pinza desde su posición de izaje. Este punto es de cuidado ya que desde este punto el eje central realiza los movimientos sutiles que liberan a la pinza del mecanismo de traba para tomar el bloque.

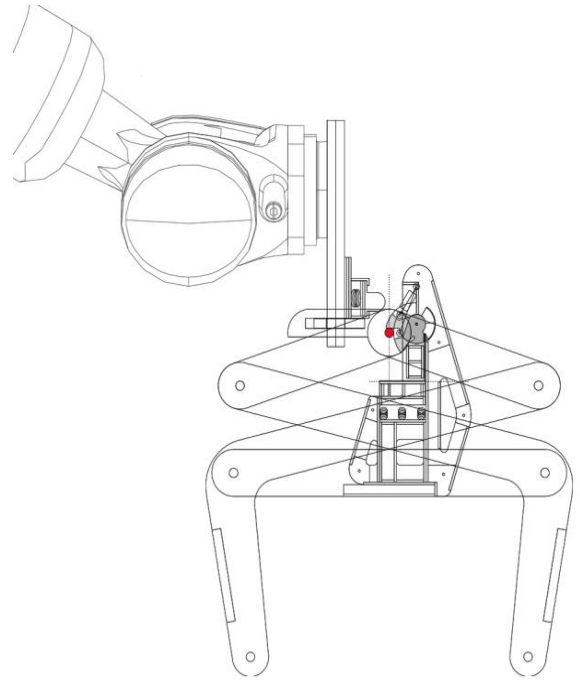

Figura 3: Plano de interior de pinza diseñada con sistema automático de traba de accionamiento mecánico, conectada al flange del robot. Elaboración propia.

3.- Posición B: Coordenadas del TCP al momento de llegar con el bloque a su posición final. Este punto igual es de cuidado ya que desde este acá el eje central realiza los movimientos tomarse de la traba y liberar el bloque.

4.- Altura máxima levantamiento en dirección "Z" que alcanza el robot con la herramienta utilizada. De esta altura dependerá el alto de la enfierradura de refuerzo a utilizar en la construcción de un muro o pilar.

5.- Radio de operabilidad del robot. La distancia máxima entre el centro del robot y punto de izaje de bloques; y el centro del robot y ubicación final del bloque. De acuerdo con las recomendaciones del simulador KUKA PRC el robot tiene un mejor desempeño en maniobras realizadas entre una franja radial que va desde los $1200 \mathrm{~mm}$. a $18000 \mathrm{~mm}$. de distancia, siendo ideal trabajar a $1500 \mathrm{~mm}$., para asegurar una altura máxima de alcance, por lo tanto, estas distancias se establecen como radio de operabilidad factible del robot.

Una vez teniendo la programación, se prepara el archivo de testeo, un archivo de texto en formato .SRC que se inserta a través de un pendrive a la memoria del robot, para que lo lea. El robot, por protocolo, es manipulado por el operador, quien está capacitado para mover el robot de forma manual en caso de cualquier percance, instalar las herramientas que el robot requiera y verifique que la programación es factible de realizar en terreno. El trabajo remoto en este punto permite que el programador pueda enviar el archivo a través de internet para que se ejecute, y a su vez, mantenga interacción con el operador para la retroalimentar la programación por videollamada. 


\section{RESULTADOS}

De acuerdo con los resultados ergonómicos se estable la idoneidad del sistema constructivo estudiado para abordad la integración robótica en los procesos de construcción, logrando sistematizar el proceso de modelado, programación de trayectorias, elaboración de herramienta y pruebas de movimientos para el montaje de estos.

\section{1.- DEL ESTUDIO ERGONÓMICO}

Según lo observado, durante el período de manipulación de la carga se evidencia sobrecarga postural en las siguientes acciones:

Al momento de tomar los bloques desde el piso y dejarlos sobre el montacargas, existe inclinación de tronco por la tomada de la carga que se encuentra al nivel de rodillas y en el caso de un trabajador por debajo de ellas, lo que se observa en la figura 5:

- En el momento de alzar la carga, lo hacen con una mano y acomodan con la otra en forma de pinza, lo que genera torsión y flexión de tronco y sobrecarga de las extremidades superiores y la zona lumbar, que es una consecuencia de una asimetría postural, generada por la naturaleza de la carga, lo que se observa en la figura 6 y 7 :

- La instancia más crítica ocurre durante el ensamble superior de los bloques en la línea de altura, ya que deben manipularlos por sobre los hombros en una posición sin sustentación equilibrada que permita la correcta interacción de los brazos y las secciones que se van armando, se observa un sobre esfuerzo en los antebrazos y muñecas y hombros provocado por la distancia entre los brazos y el centro de gravedad de la carga, lo que se observa en la figura 8 y 9 .

Según el resultado de la aplicación de la metodología Mac, la tarea se encuentra en una condición de riesgo Alto: (rojo). Se requiere introducir mejoras pronto. Esta situación podría exponer a riesgo de lesiones a los trabajadores. Cabe destacar que el peso de cada bloque es de $25 \mathrm{~kg}$ y sus medidas son $20 \times 120 \times 40$ $\mathrm{cm}$, en cuanto a peso cumple con la norma. Son las posiciones que adopta el cuerpo en la interacción con la carga las que generan la desviación ergonómica. (Manual handling assessment charts, HSE. 2003).

Carga Bioenergética: Índice de Frecuencia cardiaca y análisis fisiológico: Se efectuó una muestra de presión arterial y latidos por minuto ante y después de la tarea a los dos trabajadores que manipulaban los pesos, lo que arrojó un resultado en promedio de: Antes de la tarea: 73 latidos cardiacos por minuto, con una presión arterial sistólica: 122 ( $\mathrm{mm} \mathrm{HG}$ ) y diastólica de 79 (mm $H G)$.
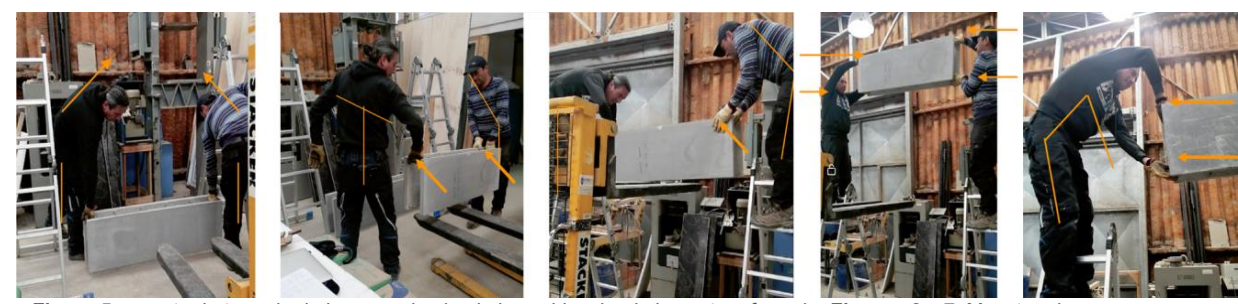

Figura 5: muestra la tomada de la carga desde el piso evidenciando la postura forzada. Figuras 6 y 7 : Muestran la manera en que toma la carga al momento de tomarla y levantarla con el cuerpo inclinado. Figuras 8 y 9: Muestran la restricción corporal generada por la manipulación de la carga y brazos alejados del cuerpo. Elaboración propia.
Después de la tarea: 85 latidos cardiacos por minuto, con una presión arterial sistólica: 137 (mm HG) y diastólica de 90 ( $\mathrm{mm} \mathrm{HG)}$. Lo que indica que la tarea es de un esfuerzo moderado.

Figura 4: Se muestran los principales movimientos de carga y movimientos corporales que deben ser apoyados por el robot. Elaboración propia.

Se recomienda incorporar ayudas mecánicas para el izaje de carga e instalación en la estructura vertical, plataformas de alcance de alturas y acercamiento de la carga para los trabajadores, evaluar la posibilidad de rotar las funciones y de incorporar asas o cavidades a los bloques que permitan una correcta manipulación. (Apud et al 2009)

Generar un protocolo de pasos de armado y manipulación, que contemple el distanciamiento entre trabajadores que interactúan con los bloques y entre sí.

Cabe destacar que todos los hallazgos encontrados y respaldados por este estudio, pueden ser superados con la implementación del proceso programado (automatizado) de instalación en serie de bloques en vertical sumando secciones horizontales en función de formar un muro continuo. Estos movimientos secuenciales, se definen a partir del análisis de movimiento efectuado a los trabajadores y las partes del cuerpo más comprometidas en la ejecución de la tarea que era el izaje vertical de la carga y la instalación en la fila de bloques. Se logra establecer que este proceso muestra una ventaja tecnológica aplicada en un método que hasta hoy es realizado de forma manual por trabajadores, en directa relación con la capacidad de rendimiento al ejercer las (volumen) de los bloques. (Barceló Fernández, 2018)

Respecto a las medidas de distanciamiento social en el trabajo, para este caso la longitud del bloque es de $1.20 \mathrm{~m}$, lo que agregado la separación de los brazos hace que se cumpla con la norma establecida en los nuevos protocolos. Además, con la implementación de una herramienta robotizada de operación remota, hace que exista una reducción de personal presencial en las áreas de trabajo de interior y exterior en obras en construcción, disminuyendo la posibilidad de contagio directo de enfermedades. labores, condicionadas por la expresión material y formal 

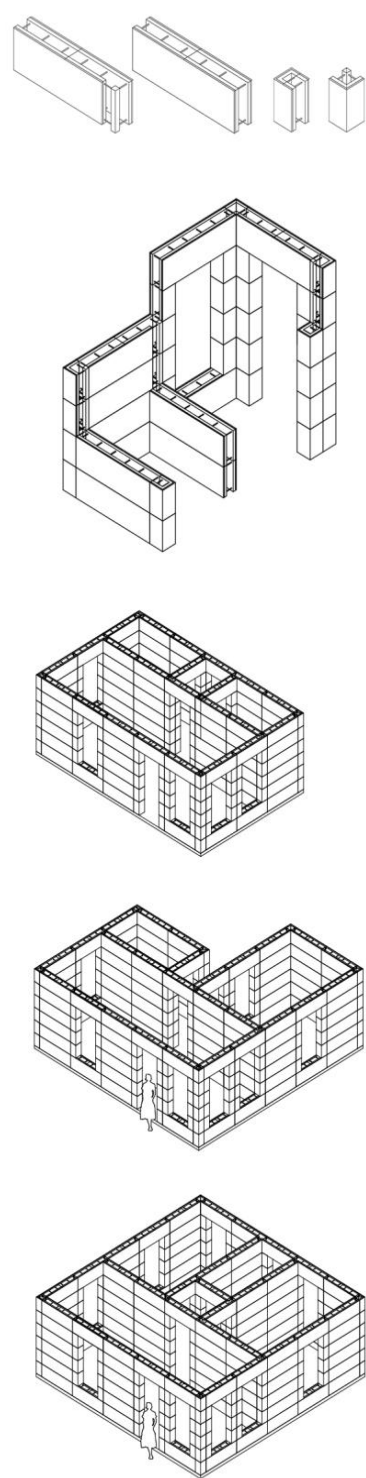

Figura 10: Modelación BIM para familias de bloques y configuraciones básicas de estas. Elaboración propia.

\section{2.- PROYECTO BIM}

Cuatro familias de elementos son una cantidad mínimas para la creación de espacios habitables: un tipo de bloque base que sirva para muros y vigas, de $120 \mathrm{~cm}$. de ancho; un bloque para intersecciones con muros interiores de $120 \mathrm{~cm}$ de ancho; un bloque de esquina y uno de terminación para cerrar bordes de muros. de ancho variable de acuerdo con requerimientos térmicos (figura 10). Cada una de las piezas corresponden a una nueva familia Revit la cual se inserta en el modelo del proyecto como componente. La interacción entre estas familias de bloques logra la continuidad interior necesaria que requiere el sistema constructivo para ser moldaje. Pensando en la optimización de la construcción robótica los bloques se diseñan para funcionar a gravedad configurando muros, columnas, puertas y ventanas mediante bloques sobrepuestos. (figura 10). Las medidas estandarizadas de este sistema permiten modular ejes cada $1.20 \mathrm{~m}$., creando espacialidades interiores con altura habitable mínima de $2,0 \mathrm{~m}$. bajo dinteles y $2,4 \mathrm{~m}$ de piso a cielo con luces interiores que varian de $1,2 \mathrm{~m}$. en 1,2m. (descontándose un espesor de bloque en muros de interior).

\section{3.- LA PINZA DE IZAJE}

Los referentes de observación para crear la herramienta que opera el robot fueron las pinzas de izaje tipo tijeras, utilizadas comúnmente para levantar pesados bloques de roca u hormigón como soleras o protecciones carreteras, esta pinza tiene la particularidad de cerrar automáticamente gracias a la fuerza de gravedad, mientras más pesado sea el bloque a levantar, más fuerte es la presión que ejerce al tomarlo, haciendo que esta maniobra de levantamiento sea un acto seguro. Sin embargo, es necesario tener en cuenta que su capacidad de carga dependerá en gran medida del material con la cual se encuentre construida.

Entonces, teniendo como material disponible para la confección de la pinza una placa de madera terciada de $12 \mathrm{~mm}$. es que se piensa en una estructura que permita abarcar la mayor cantidad de superficie de contacto con el bloque y de esta forma distribuir de mejor manera el peso del bloque hacia el flange; que tenga un punto fijo que pueda conectarse al flange del robot; y por otro lado, que tenga en cuenta un sistema de traba que permita mantener la pinza abierta para soltar el bloque una vez llegue a su posición final.

Teniendo en cuenta estos pie forzados, es que se pensó en una pinza compuesta por 2 tijeras y unidas por placas de madera en la base y en sus laterales a modo de crear una especie de "garra" de unos $20 \mathrm{~cm}$ de ancho, cuyas piezas se articulan a través de barras de acero roscado de $1 / 2$ "de espesor, sostenidos con golillas y tuercas, hasta unirse en su parte superior en un único centro fijo que conecta al flange del robot. El peso total de ésta es $6,514 \mathrm{~kg}$. (Figura 11). El sistema de traba consta de un mecanismo de accionamiento que aprovecha 2 instancias de la pinza, la parte estática (o fija) y la parte dinámica (o móvil), haciendo que la parte estática se enganche a la parte dinámica cuando se requiera dejar la pinza abierta. 


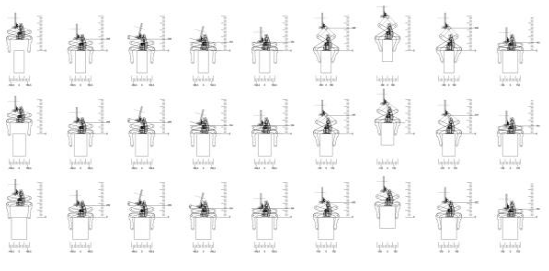

Figura 11: Secuencia de movimientos de la pinza para accionar y desactivar el sistema mecánico de traba. Elaboración propia.

\section{4.- LA PROGRMACIÓN DE LOS MOVIMIENTOS.}

Para crear un recorrido para el robot utilizamos principalmente 2 tipos de movimientos, el movimiento lineal, (LIN) donde el robot mantiene la posición del flange en todo su trayecto. Y el movimiento punto a punto (PTP), donde el robot realiza el movimiento de la forma más eficiente, pero sin necesidad de mantener la posición del flange en su trayecto. La herramienta que utilizamos se posiciona en el plano XY por lo que trabajamos con esa configuración inicial. Primero se posiciona la herramienta para tomar los bloques en el punto de izaje A, luego se llega a la máxima altura, manteniéndola se traslada sobre su siguiente posición y baja el bloque hasta ubicarse en el punto B. y dejar el bloque. A esta programación base se insertan las coordenadas de posicionamiento, se considera la altura de base del robot $(+57,5$ en este caso) y la adecuación de movimientos que accionan la pinza para que el robot la opere en el montaje.

\section{5- TESTEOS}

El primer testeo se realizó sacando coordenadas de posición A, B y altura máxima, dejando un espacio libre bajo el bloque levantado de $1,9 \mathrm{~m}$. En el segundo testeo se optó por hacer un cambio de cuadrante de trabajo en la trayectoria, a modo que los trabajadores puedan abastecer de bloques desde un área segura mientras el robot esté en movimiento. El tercer testeo incluye el accionamiento de la traba por los movimientos del robot. Las velocidades de testeos se realizan en modo T01, modo de prueba que por seguridad reduce la velocidad del robot al $10 \%(0.2 \mathrm{~m} / \mathrm{s})$, a pesar de esto, la tarea de izaje debe mantener una velocidad reducida debido a la inercia que provoca el peso del bloque en el aire.

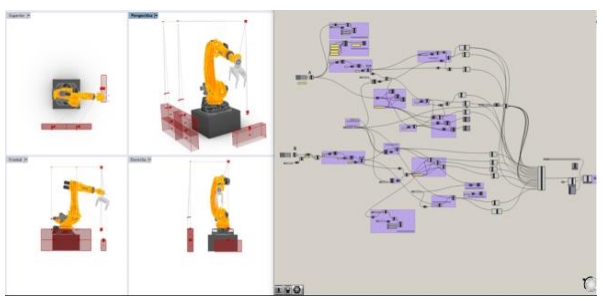

Figura 14: Programación de simulación robótica con software Rhinoceros + Grasshopper y plugin KUKA PRC. Elaboración propia.

La aceleración también dependerá de evitar sobrerevolucionar los ejes de movimientos del robot, mientras nuestra posición sea más cercana a 0 en las coordenadas $\mathrm{X}$ e $\mathrm{Y}$, mayores serán las revoluciones del eje 4 al bajar de forma directa.

Tabla 3. Coordenadas de movimientos de testeos

\begin{tabular}{|c|c|c|c|c|c|}
\hline Testeo & $\begin{array}{l}\text { Plano } \\
\text { A }\end{array}$ & $\begin{array}{l}\text { Posición } \\
\text { A }\{x, y, z\}\end{array}$ & $\begin{array}{l}\text { Plano } \\
\text { B }\end{array}$ & $\begin{array}{l}\text { Posición } \\
\text { B }\{x, y, z\}\end{array}$ & $\begin{array}{l}\text { Altura } \\
\text { máx. } \\
\text { TCP } \\
\text { (mm.) }\end{array}$ \\
\hline T01 & YZ 180 & $\begin{array}{c}658.15 \\
-1843.94 \\
136.28\}\end{array}$ & YZ 180 & $\begin{array}{c}\{-690.67 \\
-1885.53 \\
133.06\}\end{array}$ & 2271.63 \\
\hline T02 & $X Y \quad 0^{\circ}$ & $\begin{array}{c}\{1500 \\
0,136\}\end{array}$ & $\begin{array}{r}X Y 90^{\circ} \\
13\end{array}$ & $\begin{array}{c}\{-272 \\
-1478 \\
36 / 536 / 936\}\end{array}$ & 1771.35 \\
\hline T03 & $X Y$ & $\begin{array}{r}\{1414 \\
0,201\}\end{array}$ & $\begin{array}{r}X Y 90^{\circ} \\
201\end{array}$ & $\begin{array}{l}\{-586 / 414 \\
-1200 \\
/ 601 / 201 / 601\end{array}$ & 2501.00 \\
\hline
\end{tabular}

Tabla 4. Velocidades, longitud recorridos y tiempo de ejecución.

\begin{tabular}{|c|c|c|c|c|c|}
\hline Testeo & $\begin{array}{l}\text { Vel. } \\
\text { config. }\end{array}$ & $\begin{array}{l}\text { Vel. } \\
\text { robot }\end{array}$ & $\begin{array}{l}\text { Cantidad } \\
\text { Bloques }\end{array}$ & $\begin{array}{l}\text { Recorrido } \\
\text { (mm.) }\end{array}$ & $\begin{array}{l}\text { Tiempo } \\
\text { (s) }\end{array}$ \\
\hline $\bar{T}$ T01 & $\begin{array}{l}\text { PTP } 45 \% \\
\text { LIN 0,5 } / 5 / \mathrm{s}\end{array}$ & $\begin{array}{l}\mathrm{T}-1 \\
10 \%\end{array}$ & 1 & $13.384,58 \mathrm{~mm}$. & 170 \\
\hline T02 & $\begin{array}{l}\text { PTP } 45 \% \\
\text { LIN } 0,5 \mathrm{~m} / \mathrm{s}\end{array}$ & $\begin{array}{l}\text { T-1 } \\
10 \%\end{array}$ & 3 & $31.337,92 \mathrm{~mm}$. & . 259 \\
\hline T03 & $\begin{array}{l}\text { PTP } 45 \% \\
\text { LIN } 0,5 \mathrm{~m} / \mathrm{s}\end{array}$ & $\begin{array}{l}\mathrm{T}-1 \\
10 \%\end{array}$ & 4 & $49.875,66 \mathrm{~mm}$ & . 1273 \\
\hline
\end{tabular}

Nota: testeos 1 y 2 realizado con bloque reales de 1,3m. Testeo 3 , con bloques de maqueta de $1 \mathrm{~m}$. de ancho.

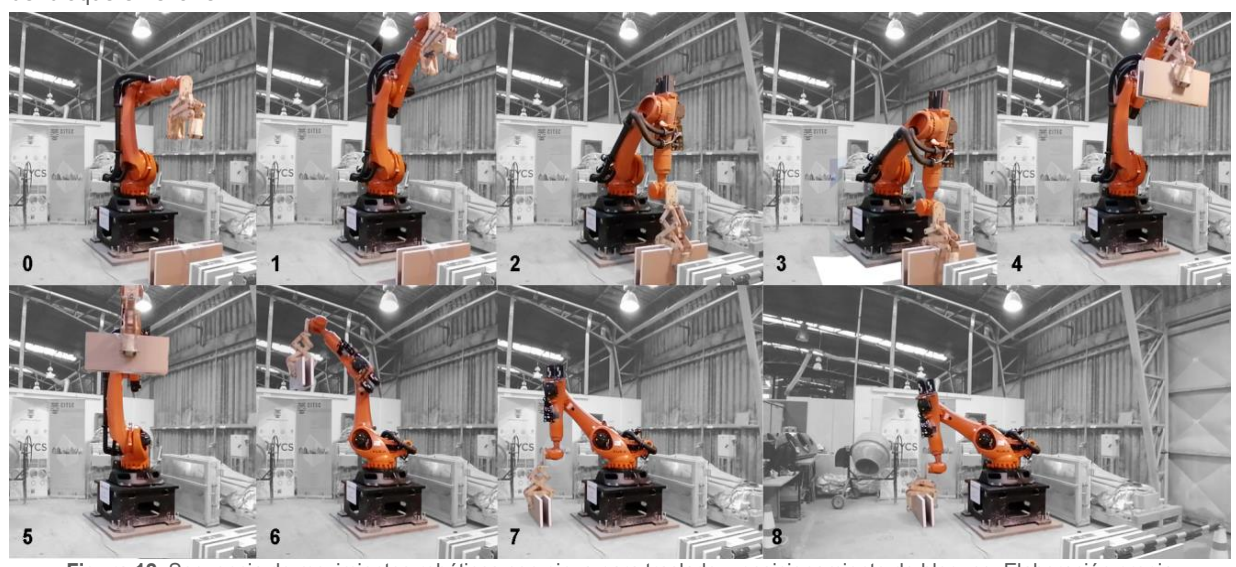

Figura 12: Secuencia de movimientos robóticos con pinza para traslado y posicionamiento de bloques. Elaboración propia. 


\section{DISCUSIÓN}

El estudio permite poner en problemática la utilidad de la robótica en actividades humanas, como una oportunidad de transformar procesos constructivos e incorporar la faena a distancia en sociedades susceptibles a pandemias y necesitadas de distanciamiento social. También se reconoce la necesidad de adaptar las herramientas tecnológicas creadas en países avanzados a las realidades locales, combinando capacidades y focalizando los desarrollos.

En el diseño arquitectónico se advierte la incidencia de un sistema prefabricado más eficiente, pero también los requerimientos de faenas en altura, que generan riesgos laborales. De modo que la consideración de seguridad en la obra y eficacia en el montaje debe considerarse en la planificación del edificio.

El trabajo avanza en una automatización productiva de la construcción, reduciendo el personal presente en obra, lo que implica adaptar las faenas, para desarrollar las competencias necesarias de la fuerza laboral y proveer entornos más seguros en la construcción, dotando de las herramientas requeridas a las labores constructivas y adaptando al diseño arquitectónico a la modulación de montaje, permitiendo un flujo de trabajo más directo entre diseño y obra. Lo que brinda una nueva perspectiva a la arquitectura y construcción como una integración de capacidades y requerimientos, con condiciones de bienestar en todos sus aspectos, mediante la combinación de esfuerzos humanos y digitales.

\section{AGRADECIMIENTOS}

Nuestros agradecimientos a CIPICS_UBB por patrocinio de instalaciones y Juan Guillermo Sandoval, CITEC_UBB por su apoyo en la operación del robot.

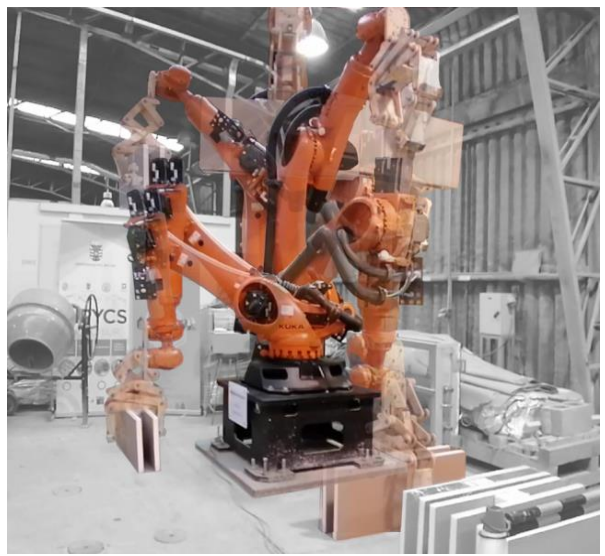

Figura 13: Movimientos del robot mostrados simultáneamente. Elaboración propia.

\section{REFERENCIAS}

CORFO (2015) Productividad y Construcción Sustentable. Retrieved from

http://www.agendaproductividad.cl/wpcontent/uploads/sites/2 2/2014/10/PPT_Programa_Estrategico_Construccion_Susten table.pdf

Baldwin, A., Poon, C.-S., Shen, L.-Y., Austin, S., \& Wong, I. (2008). Modelling design information to evaluate prefabricated and pre-cast design solutions for reducing construction waste in high rise residential buildings. Automation in Construction, 17(3), 333-341. https://doi.org/10.1016/j.autcon.2007.05.013

Jaillon, L., \& Poon, C.-S. (2008) Sustainable construction aspects of using prefabrication in dense urban environment: a Hong Kong case study. Construction Management and Economics, 26 (9), 953-966.

De Sutter, S., Remy, O., Tysmans, T., \& Wastiels, J. (2014). Development and experimental validation of a lightweight Stay-in-Place composite formwork for concrete beams. Construction and Building Materials, 63, 33-39.

Bock, T. (2015) Construction Robotics enabling Innovative Disruption and Social Supportability, 2015 Proceedings of the 32st ISARC, Oulu, Finland [18] Gambao, E., \& Balaguer, C. 2002. Robotics and automation in construction. IEEE Robotics and Automation Magazine (Vol. 9).

Lim S., Buswell R.A., Valentine P.J., Piker D., Austin S.A., De Kestelier X. (2016), Modelling curved-layered printing paths for fabricating large-scale construction components, Addit. Manuf. 12, pp. 216-230

Sousa J.P. (2017), Robotic Technologies for Non-Standard Design and Construction in Architecture, Nexus Network Journal, 19-1, pp 73-83

Davtalab O., Kazemian A., Khoshnevis B (2018) Perspectives on a BIM-integrated software platform for robotic construction through Contour Crafting, Autom. Constr. 89, pp. 13-23

Reinhardt,Dagmar., Sunders, Rob., Burry, Jane. (2016). Robotic Fabrication in Arqchitectura, Art And Desing. London: Springer.

García, R., Pérez, A., Pulido, J., Ulloa, P., Sepúlveda J., Forcael, E. (2019). "Robotic assembly of stay-in-place concrete blocks, .Sustainable Built Environment 2019. Temuco: SBE Conference.

Reyes, B. Claudio. (2019). Informe Anual Estadísticas de Accidentabilidad. Santiago: Superintendencia de Seguridad Social. Chile.

Superintendencia de Seguridad Social. (2017). Informe Anual Estadísticas de Accidentabilidad. Santiago: Superintendencia de Seguridad Social. Chile.

Abarca,A.,(2005).El Factor Humano y su incidencia en el proyecto de construcción. México. UAM.

Manual handling assessment charts (the MAC tool)(2003)Londres, Health and Safety Executive. UK.

Barceló Fernandez, J., (2018). Incidencia del comportamiento humano en los accidentes del trabajo. Barcelona, Universidad de Barcelona. España

Carrasco, A., Donari, J., (2016). Accidentabilidad laboral: análisis de las causas más recurrentes y factores intervinientes. Santiago.Dirección del Trabajo. Chile.

K Chu,D., (2020). Distanciamiento físico, mascarillas y protección ocular para prevenir la transmisión de persona a persona de SARS-CoV-2 y COVID-19: en una revisión sistemática de metaanálisis. Hamilton. Departamento de Medicina McMaster University. UK

Achs (2016) .Trabajos en altura. Recuperada desde https://www.achs.cl/portal/Empresas/fichas/Paginas/Trabajos -en-altura.aspx 